\title{
Modeling Progressive Failure of Bonded Joints Using a Single Joint Finite Element
}

\author{
Scott E. Stapleton ${ }^{1}$ and Anthony M. Waas ${ }^{2}$ \\ University of Michigan, Ann Arbor, MI, 48109, USA \\ and \\ Brett A. Bednarcyk ${ }^{3}$ \\ NASA Glenn Research Center, Cleveland, OH, 44135, USA
}

\begin{abstract}
Enhanced finite elements are elements with an embedded analytical solution which can capture detailed local fields, enabling more efficient, mesh independent finite element analysis. In the present study, an enhanced finite element is applied to generate a general framework capable of modeling an array of joint types. The joint field equations are derived using the principle of minimum potential energy, and the resulting solutions for the displacement fields are used to generate shape functions and a stiffness matrix for a single joint finite element. This single finite element thus captures the detailed stress and strain fields within the bonded joint, but it can function within a broader structural finite element model. The costs associated with a fine mesh of the joint can thus be avoided while still obtaining a detailed solution for the joint. Additionally, the capability to model non-linear adhesive constitutive behavior has been included within the method, and progressive failure of the adhesive can be modeled by using a strain-based failure criteria and re-sizing the joint as the adhesive fails. Results of the model compare favorably with experimental and finite element results.
\end{abstract}

\section{Introduction}

T $\mathrm{N}$ the aerospace industry, fiber reinforced polymer matrix composites (FRPCs) are gaining increasing use and attention because of their high strength to weight ratios, among other factors. FRPC joints perform much better with adhesive bonding rather than bolting or riveting because of their quasi-brittle nature ${ }^{1}$ and the ability of the bond

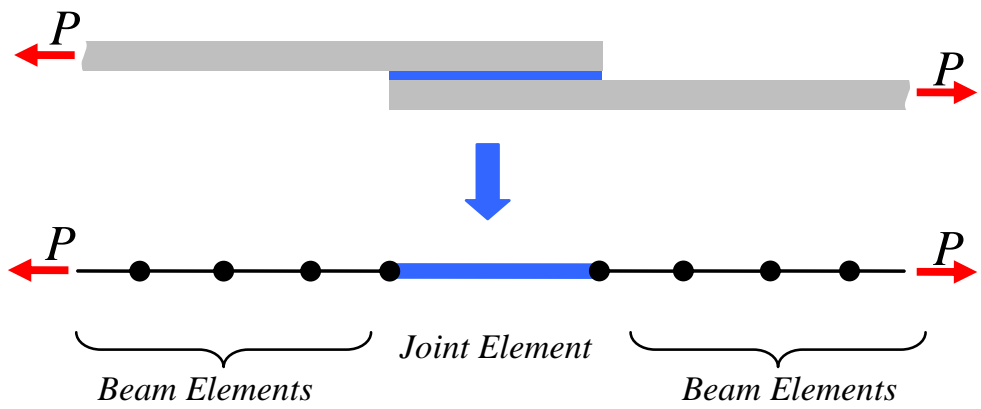

Figure 1. The present study replaces a complex single lap joint mesh with a single joint element. to spread the load over a larger area leading to a lessening of the stress concentration $^{2}$. Therefore, accurate analysis of adhesively bonded joints is becoming more critical than ever.

Adhesive joints have traditionally been analyzed using two methods: analytical models and finite element analysis ${ }^{3}$. Analytical methods have been utilized to extract efficient closed-form solutions for adhesive single lap joint stresses. Classical formulas have been introduced by Volkerson ${ }^{4}$,

\footnotetext{
${ }^{1}$ Graduate Student Research Assistant, Department of Aerospace Engineering. Email: sstaple@umich.edu

${ }^{2}$ Professor, Department of Aerospace Engineering. Associate Fellow, AIAA. Email: dcw@umich.edu

${ }^{3}$ Material Research Engineer, 21000 Brookpark Rd. Senior Member, AIAA. Email: Brett.A.Bednarcyk@NASA.gov 
Goland and Reissner ${ }^{5}$ and Hart-Smith ${ }^{6}$. More recently, refined analytical studies, carried out by Mortensen and Thomsen ${ }^{7}$ and Delale et al., ${ }^{8}$ have proven to be quite accurate in predicting stresses within adhesive joints. However, analytical methods are often limited by geometric assumptions used to obtain a closed form solution and are not as useful to designers for compiling vehicle-scale models that may contain multiple joints. Finite element analyses are widely utilized in industry, and can be used to assess joints with a wide variety of geometries and loading conditions. However, these methods can suffer from mesh dependence and a lack of efficiency, which is especially crippling for initial sizing analysis and full vehicle-scale models ${ }^{9}$. Therefore, a need exists to develop predictive tools for bonded joints that can be seamlessly coupled with large scale structural analyses without adding major computational costs. Such tools can be used to make quick mesh-independent assessments of bonded composite joints. Currently, such a capability is lacking, and joint assessment is typically performed late in the design cycle when structural changes that can lower the weight are much more difficult and expensive.

Gustafson and Waas ${ }^{3}$ have merged analytical and finite element methods in order to perform efficient, mesh independent finite element analysis of double lap joints to use for initial design and macroscopic vehicle modeling. Analytical models were embedded into a single finite element with minimal analyst input. Stapleton and Waas ${ }^{10}$ extended this method to single lap joints (Fig. 1), which are more complex due to the eccentricity of the axial load path. The adherends were treated as Euler-Bernoulli beams, and the adhesive was modeled as a bed of normal and shear springs. The current study extends the method to include a nonlinear constitutive model for the adhesive in conjunction with inputs to cohesive zone finite element modeling ${ }^{11,12}$ or a curve-fit to experimental test data. Additionally, a strain-based failure criterion is utilized to track damage in the adhesive and the joint element and adjacent beam elements are re-sized to account for the failed adhesive. The entire joint can then be replaced by a single joint finite element, while the remaining structure (outside the joint) is modeled using standard structural elements, for instance beam elements (Fig. 1). As a result, failure in the adhesive is built into the joint element.

\section{Analytical Formulation}

The joint element with adhesive failure capabilities is an extension of the linear elastic joint element derived elsewhere (Stapleton and $\mathrm{Waas}^{10}$ ). The main difference is that this model now has the capability of handling adhesives with non-linear constitutive behavior and has a failure criteria implemented within the model. A flow chart of the joint element implementation is shown in Fig. 2.

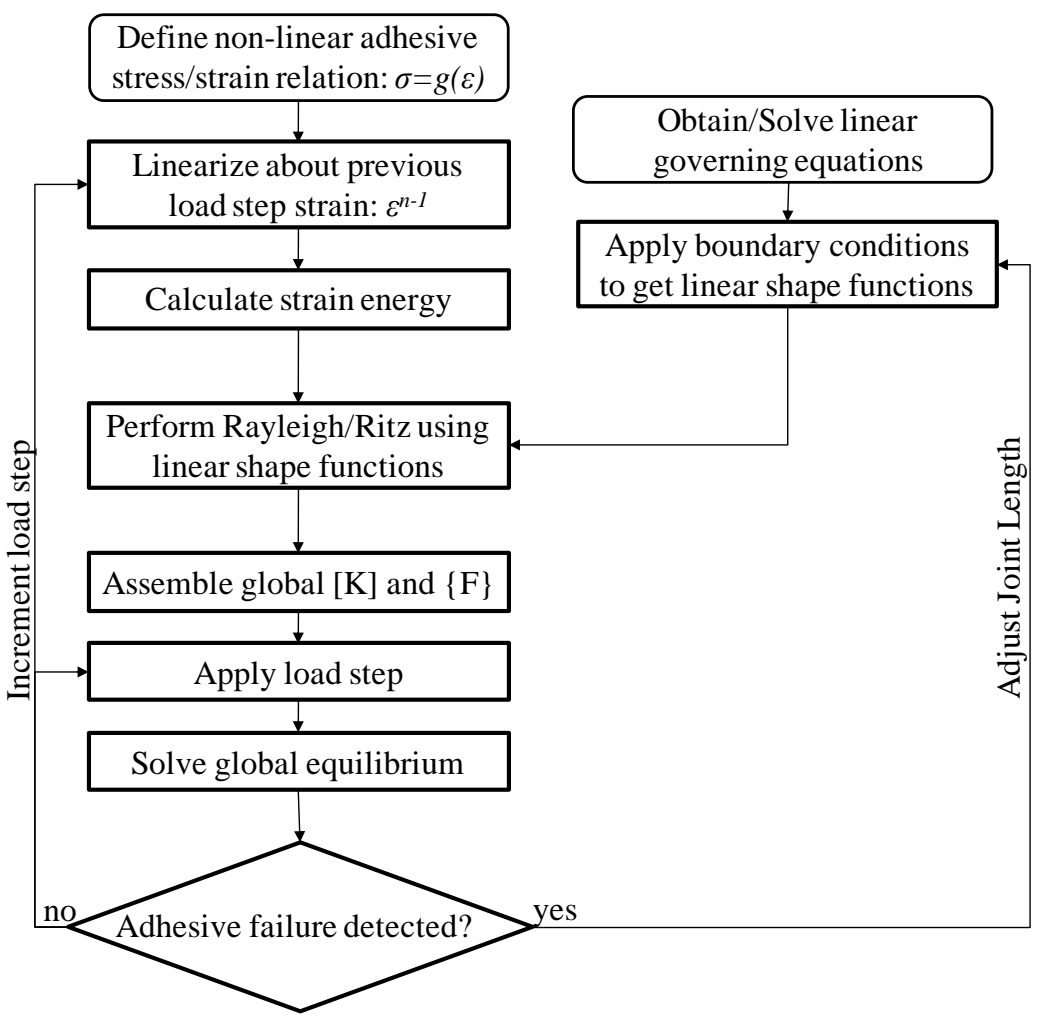

Figure 2. Flow chart of the joint element with adhesive progressive failure.

American Institute of Aeronautics and Astronautics 092407 
The individual steps will be expanded upon in the following sections, but the basic layout consists of solving the linear problem first to obtain shape functions, defining a non-linear stress/strain relation for the adhesive and linearizing this relation at each load increment, calculating the joint stiffness matrix and force vector using the linear shape functions and strain energy for the non-linear case, and solving the resulting equations. Furthermore, the adhesive is checked for failure, and in the case of adhesive failure, the length of the joint element decreases to account for failed adhesive.

\section{A. Obtain Linear Shape Functions}

In order to model nonlinear adhesive in a joint element, the shape functions are obtained from the case of a joint with a linear adhesive. To do this, the adhesive and adherends were assumed to be linearly elastic. Figure 3 illustrates the required geometric parameters and material properties of a single lap joint. The width of the joint in the y-direction is $b$. The subscripts 1,2 , and $a$ denote a variable associated with adherend 1,2, or the adhesive.

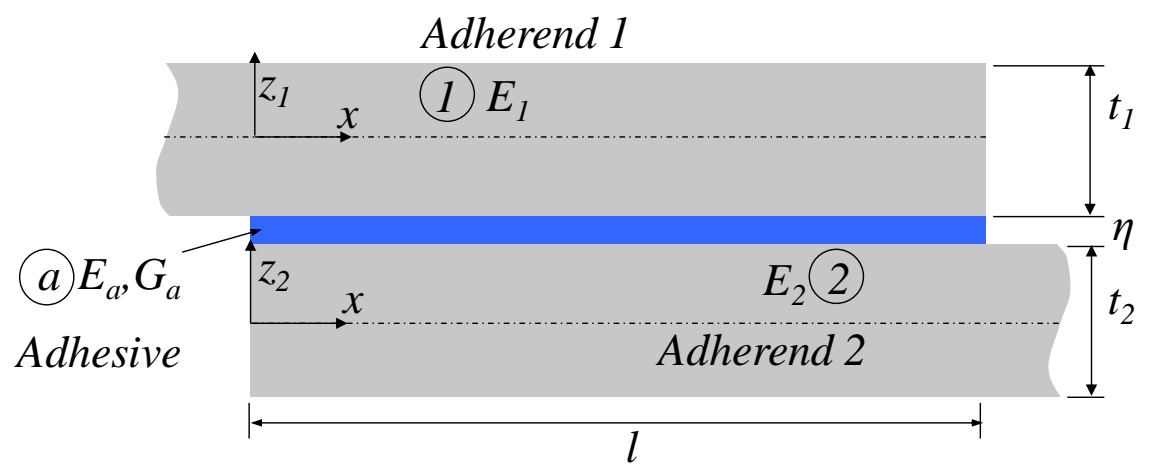

Figure 3. Required geometric and material parameters for overlap region of a single lap joint.

Assuming the adherends behave like Euler-Bernoulli beams, the strain energy of the joint, $U$, is written as:

$$
U_{\text {Joint }}=\frac{1}{2} \int_{V_{1}} \sigma_{1 x} \epsilon_{1 x} d V+\frac{1}{2} \int_{V_{2}} \sigma_{2 x} \epsilon_{2 x} \mathbb{d} V+\frac{1}{2} \int_{V_{a}}\left(\sigma_{a z} \epsilon_{a z}+\tau_{a x z} \gamma_{a x z}\right) d V
$$

where $\sigma_{i x}$ and $\epsilon_{i x}$ represent the normal stress and strain in material $i$ ( 1 or 2 for the adherends) in the $x$ - direction, $\sigma_{a z}$ and $\epsilon_{a z}$ are the normal stresses/strains in the adhesive in the z-direction, $\tau_{a x z}$ and $\gamma_{a x z}$ represent the shear stress/strain in the adhesive on the $x z$-plane, and all integrals are taken over the volume, $V_{i}$ of material $i$.

The two adherends are assumed to behave as beams, and the strain energy of the adherends can be found using standard Euler-Bernoulli beam theory. The derivation is not shown here, but the details are contained in Stapleton and Waas ${ }^{10}$.

It should be noted that many adhesive joints involving bonded FRPCs are made up of thin adherends which are relatively long in the $y$-direction, causing them to behave more like plates in cylindrical bending rather than beams. Though not done herein, to model the adherends as wide plates in cylindrical bending, it is only a simple matter of replacing the modulus of elasticity, $E_{i}$ with $E_{i} /\left(1-v_{i}^{2}\right), i=1,2$ and modeling the adhesive using plane strain, rather than plane stress assumptions.

A diagram of the notation scheme for the adhesive is shown in Fig. 4. It is assumed that the displacement varies linearly in the $z_{a}$-direction and that the adhesive and adherend are perfectly bonded at the interface. The displacements at the interface will be denoted by the subscript $i$. 


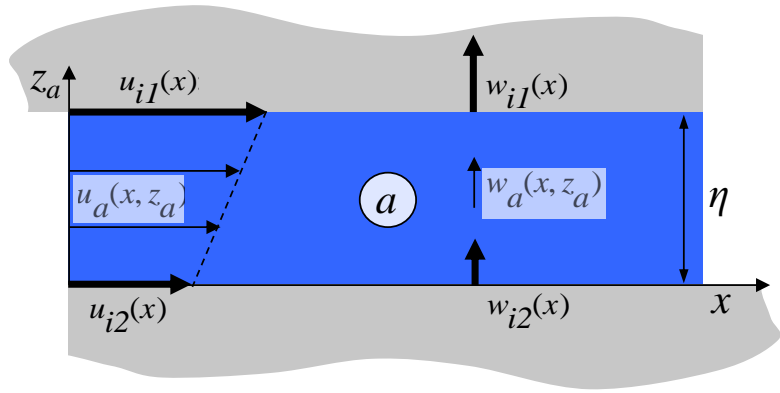

Figure 4. Geometric and material parameters for overlap region of a single lap joint.

The adhesive is modeled as a bed of linear normal and shear springs. In terms of the interface displacements, the strains in the adhesive are:

$$
\epsilon_{a z}=\frac{1}{\eta}\left(w_{i 1}(x)-w_{i 2}(x)\right)
$$

and

$$
\gamma_{a x z}=\frac{1}{\eta}\left(u_{\mathrm{i} 1}(x)-u_{\mathrm{i} 2}(x)\right)
$$

and the stresses are:

and

$$
\sigma_{a z}=E_{a} \epsilon_{a z}
$$

$$
\tau_{a x z}=G_{a} \gamma_{a x z} .
$$

The interface displacements are defined in terms of adherend centerline displacements using Euler-Bernoulli beam theory:

$$
\begin{gathered}
u_{\mathrm{i} 1}(x)=u_{1}(x)+\frac{t_{1}}{2}\left(\frac{\mathbb{d} w_{1}(x)}{\mathbb{d} x}\right), u_{\mathrm{i} 2}(x)=u_{2}(x)-\frac{t_{2}}{2}\left(\frac{\mathbb{d} w_{2}(x)}{\mathbb{d} x}\right), \\
w_{\mathrm{i} 1}(x)=w_{1}(x), \text { and } w_{\mathrm{i} 2}(x)=w_{2}(x) .
\end{gathered}
$$

Using the principle of stationarity of potential energy, four fully coupled governing equilibrium differential equations are obtained from the energy expression in Eq. (1). Of the four governing equations, two correspond to the axial equilibrium, while two equations correspond to the transverse equilibrium. The axial displacement equilibrium equations contain second order derivatives, while the transverse displacement equations have fourth order derivatives. The order of these equations can be reduced and assembled into a system of first order constant coefficient homogeneous ordinary differential equations of the form:

$$
\{u\}_{, \mathrm{x}}=[A]\{u\}
$$

where

$$
\{u\}=\left[\begin{array}{llllllllllll}
u_{1} & u_{1, x} & w_{1} & w_{1, x} & w_{1, x x} & w_{1, x x x} & u_{2} & u_{2, x} & w_{2} & w_{2, x} & w_{2, x x} & w_{2, x x x}
\end{array}\right]^{\mathrm{T}}
$$

and 


$$
[A]=\left[\begin{array}{cccccccccccc}
0 & 1 & 0 & 0 & 0 & 0 & 0 & 0 & 0 & 0 & 0 & 0 \\
\lambda_{1} & 0 & 0 & \frac{1}{2} t_{1} \lambda_{1} & 0 & 0 & -\lambda_{1} & 0 & 0 & \frac{1}{2} t_{2} \lambda_{1} & 0 & 0 \\
0 & 0 & 0 & 1 & 0 & 0 & 0 & 0 & 0 & 0 & 0 & 0 \\
0 & 0 & 0 & 0 & 1 & 0 & 0 & 0 & 0 & 0 & 0 & 0 \\
0 & 0 & 0 & 0 & 0 & 1 & 0 & 0 & 0 & 0 & 0 & 0 \\
0 & \frac{1}{2} t_{1} \Lambda_{1} & -\Omega_{1} & 0 & \frac{1}{4} t_{1}{ }^{2} \Lambda_{1} & 0 & 0 & -\frac{1}{2} t_{1} \Lambda_{1} & \Omega_{1} & 0 & \frac{1}{4} t_{1} \Lambda_{1} t_{2} & 0 \\
0 & 0 & 0 & 0 & 0 & 0 & 0 & 1 & 0 & 0 & 0 & 0 \\
-\lambda & 0 & 0 & -\frac{1}{2} t_{1} \lambda_{2} & 0 & 0 & \lambda_{2} & 0 & 0 & -\frac{1}{2} t_{2} \lambda_{2} & 0 & 0 \\
0 & 0 & 0 & 0 & 0 & 0 & 0 & 0 & 0 & 1 & 0 & 0 \\
0 & 0 & 0 & 0 & 0 & 0 & 0 & 0 & 0 & 0 & 1 & 0 \\
0 & 0 & 0 & 0 & 0 & 0 & 0 & 0 & 0 & 0 & 0 & 1 \\
0 & \frac{1}{2} t_{2} \Lambda_{2} & \Omega_{2} & 0 & \frac{1}{4} t_{2} \Lambda_{2} t_{1} & 0 & 0 & -\frac{1}{2} t_{2} \Lambda_{2} & -\Omega_{2} & 0 & \frac{1}{4} t_{2}^{2} \Lambda_{2} & 0
\end{array}\right]
$$

with

$$
\begin{gathered}
\lambda_{i}=\frac{G_{a} b}{\eta E A_{i}}, i=1,2 \\
\Lambda_{i}=\frac{G_{a} b}{\eta E I_{i}}, i=1,2 \\
\Omega_{i}=\frac{E_{a} b}{\eta E I_{i}}, i=1,2 .
\end{gathered}
$$

Inspecting the matrix $[A]$ can be helpful in determining the nature of the solution and determining the solution method. There are 12 eigenvalues of $[A]$ : two real eigenvalues, four complex eigenvalues, and six repeating eigenvalues. Therefore, the solution is made up of two exponential terms, four exponential terms multiplied by a sine or cosine, and the six repeating eigenvalues correspond to a third order polynomial found in a standard beam solution. Such a complex solution shows that merely employing standard beam shape functions to the joint problem would be inadequate in capturing the nature of the whole solution.

The system in Eq. (6) can be solved using various methods, but calculating the matrix exponential was the chosen method because numerical boundary conditions are not required to obtain a solution. The solution of the system in Eq. (6) can be written in terms of the matrix exponential, $\left[e^{A x}\right]$, as

$$
\{u\}=\left[e^{A x}\right]\{C\} .
$$

The matrix exponential can be expressed as the infinite series ${ }^{13}$

$$
\left[\mathbb{e}^{A x}\right]=\sum_{k=0}^{\infty} \frac{x^{k}}{k !}[A]^{k} .
$$

In order to get faster convergence, a method of scaling and squaring ${ }^{14}$ is employed, and the series is calculated up to a value of $k$ which yields an acceptable error, $\epsilon$. The error can be defined many ways, but the current study defined the error as the difference between the 1-norms of $\left[e^{A x}\right]$ for $k-1$ and $k$. The value of the acceptable error was set at $\epsilon=0.0001$. The next step is to solve for the vector of constants, $\{C\}$, using the boundary conditions. It is at 
this point that the methods to model a single lap joint, double cantilever beam, and bonded doubler diverge. For the current study, a double cantilever beam (DCB) will be used to validate the joint element; therefore the boundary conditions will be those of the DCB (Fig. 5). Applying prescribed displacements to the joined nodes on the left gives the following system of equations:

$$
\left[B C_{0}\right]\left\{u_{0}\right\}=\{\mathrm{q}\}
$$

where $\left\{u_{0}\right\}$ is $\{u\}$ evaluated at $x=0$,

$$
\{q\}=\left[\begin{array}{llllll}
q_{1} & q_{2} & q_{3} & q_{4} & q_{5} & q_{6}
\end{array}\right]^{T},
$$

and

$$
\left[B C_{0}\right]=\left[\begin{array}{llllllllllll}
1 & 0 & 0 & 0 & 0 & 0 & 0 & 0 & 0 & 0 & 0 & 0 \\
0 & 0 & 0 & 0 & 1 & 0 & 0 & 0 & 0 & 0 & 0 & 0 \\
0 & 0 & 0 & 0 & 0 & 1 & 0 & 0 & 0 & 0 & 0 & 0 \\
0 & 0 & 1 & 0 & 0 & 0 & 0 & 0 & 0 & 0 & 0 & 0 \\
0 & 0 & 0 & 0 & 0 & 0 & 0 & 0 & 1 & 0 & 0 & 0 \\
0 & 0 & 0 & 0 & 0 & 0 & 0 & 0 & 0 & 1 & 0 & 0
\end{array}\right] .
$$

At the right end of the joint, the nodes are not joined to any other elements and are referred to as "free nodes" (Fig. 5). At these nodes, the natural boundary conditions obtained by minimizing the strain energy (Eq. (6)) are applied. These boundary conditions enforce a traction free boundary at the ends, and there is a term for the axial force, transverse force, and moment at the free nodes.

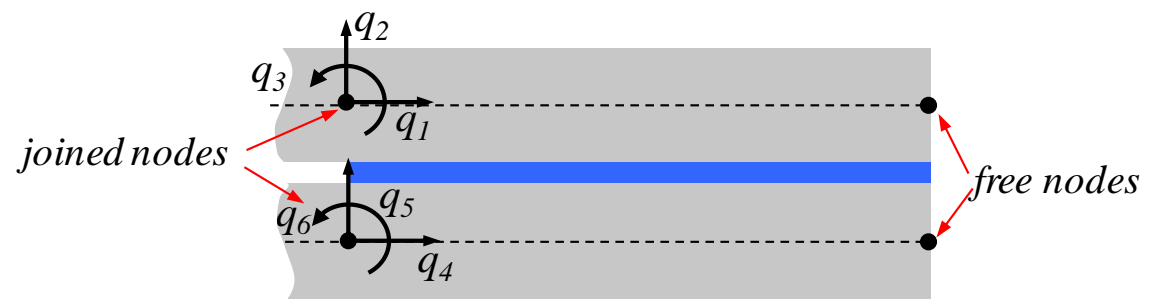

Figure 5. Boundary conditions for a DCB type joint element: prescribed nodal displacements at the joined nodes $(x=0)$ and stress free at the free nodes $(x=l)$.

The boundary conditions are applied in the following equation:

$$
\left[B C_{l}\right]\left\{u_{l}\right\}=\{0\}
$$

where $\left\{u_{l}\right\}$ is $\{u\}$ evaluated at $x=l,\{0\}$ is a vector of zeros, and

$$
\left[B C_{1}\right]=\left[\begin{array}{cccccccccccc}
0 & 1 & 0 & 0 & 0 & 0 & 0 & 0 & 0 & 0 & 0 & 0 \\
0 & 0 & 0 & 1 & 0 & 0 & 0 & 0 & 0 & 0 & 0 & 0 \\
\frac{1}{2} t_{1} \Lambda_{1} & 0 & -\frac{1}{2} t_{1} \Lambda_{1} & 0 & 0 & \frac{1}{4} t_{1}^{2} \Lambda_{1} & 0 & -1 & 0 & \frac{1}{4} t_{1} \Lambda_{1} t_{2} & 0 & 0 \\
0 & 0 & 0 & 0 & 0 & 0 & 1 & 0 & 0 & 0 & 0 & 0 \\
0 & 0 & 0 & 0 & 0 & 0 & 0 & 0 & 0 & 0 & 1 & 0 \\
\frac{1}{2} t_{2} \Lambda_{2} & 0 & -\frac{1}{2} t_{2} \Lambda_{2} & 0 & 0 & \frac{1}{4} t_{2} \Lambda_{2} t_{1} & 0 & 0 & 0 & \frac{1}{4} t_{2}^{2} \Lambda_{2} & 0 & -1
\end{array}\right] .
$$


The first and fourth rows of the matrix in Eq. (18) correspond to the axial force, the second and fifth correspond to the moment, and the third and sixth are the shear. Equation (12) is substituted into Eq. (18) and Eq. (17) and stacked on top of each other to get

$$
[\zeta]\{C\}=\left\{\begin{array}{l}
\mathrm{q} \\
0
\end{array}\right\} .
$$

where

$$
[\zeta]=\left[\begin{array}{l}
{\left[B C_{0}\right]\left[e^{A 0}\right]} \\
{\left[B C_{l}\right]\left[e^{A l}\right]}
\end{array}\right]
$$

Using this relation, one can obtain an expression for $\{C\}$ :

$$
\{C\}=[\zeta]^{-1}\left\{\begin{array}{l}
q \\
0
\end{array}\right\}
$$

The expression can be simplified further by removing the zeros from the right hand side along with six columns on the right side of $[\zeta]^{-1}$ to get $\{C\}$ in terms of the nodal degrees of freedom only. This reduced matrix will be denoted by $\left[\zeta^{\prime}\right]$. The constants are now given in terms of the nodal degrees of freedom:

$$
\{C\}=\left[\zeta^{\prime}\right]\{q\} .
$$

This relation can be inserted into Eq. (12) to get the adherend centerline displacements in terms of the nodal degrees of freedom, $q_{1-6}$ :

$$
\{u\}=[N]\{q\}
$$

where the shape functions, $[N]$, are defined as:

$$
[N]=\left[e^{A x}\right]\left[\zeta^{\prime}\right] .
$$

\section{B. Define Adhesive Stress/Strain Relation}

With the shape functions determined for a joint with a linear adhesive, the non-linear constitutive stress/strain relations of the adhesive need to be defined. This function can be based on constitutive relations, or fracture mechanics. A stress/strain relationship based on constitutive relations would take a tensile stress/strain curve with a fitting function to the data. A relation based on fracture mechanics would use a function which has its maximum stress at the mode one critical stress $\left(\sigma_{I c}\right)$ and the area under the curve would be the critical strain energy release rate of the adhesive $\left(G_{I C}\right)$. Regardless of the method used to define the relation, the adhesive stress, $\sigma_{a}$ can be written as a function of the adhesive strain, $\epsilon_{a}$ :

$$
\sigma_{a}=g\left(\epsilon_{a}\right)
$$

Although this paper refers to the normal stress and strain in the adhesive only, the same derivation holds for the shear strain/stress relation. For simplicity, the subscript $a$ will be dropped from the stress and strain symbols.

It should also be noted that this stress/strain relation assumes no permanent plasticity, but resembles non-linear elasticity. Although it is acknowledged that this relation is not representative of the actual behavior of most adhesives, this relation was found to be sufficient for the purpose of estimating failure loads to aid in initial vehicle design and scaling.

\section{Linearize Stress/Strain Relation}

To simplify calculations and avoid the need for a non-linear solver, the stress/strain relation of the adhesive is linearized about the previous strain increment. The Taylor series expansion of the stress about a strain at load step $n, \epsilon^{n}$ is: 


$$
\sigma(\epsilon)=g\left(\epsilon^{n}\right)+\frac{d g\left(\epsilon^{n}\right)}{d \epsilon}\left(\epsilon-\epsilon^{n}\right)+H O T
$$

where HOT represents higher order terms. To linearize, the higher order terms are ignored.

\section{Calculate the Adhesive Strain Energy}

The adhesive normal strain energy $U^{n+1}$ at the next load step, $n+1$, is found as the strain energy from the previous increment plus the integral of the stress as a function of strain from the previous increments to the current increment:

$$
U^{n+1}=\int_{V_{a}} \int_{\epsilon^{n}}^{\epsilon^{n+1}} \sigma(\epsilon) d \epsilon d V+U^{n} .
$$

Carrying out the inner integral gives:

$$
U^{n+1}=\int_{V_{a}}\left(\frac{1}{2} \frac{d g\left(\epsilon^{n}\right)}{d \epsilon}\left(\epsilon^{n+1}\right)^{2}+\left(g\left(\epsilon^{n}\right)-\frac{d g\left(\epsilon^{n}\right)}{d \epsilon} \epsilon^{n}\right) \epsilon^{n+1}-g\left(\epsilon^{n}\right) \epsilon^{n}-\frac{1}{2} \frac{d g\left(\epsilon^{n}\right)}{d \epsilon}\left(\epsilon^{n}\right)^{2}\right) d V+U^{n} .
$$

\section{E. Perform Rayleigh/Ritz Using Linear Adhesive Shape Functions}

To obtain the stiffness and force matrices for the joint, the shape functions derived for the linear adhesive case (Eq. 5) are used. Using Eq. (2) and (4) and the shape functions derived for the linear adhesive case, the strain in the adhesive is found in terms of the nodal displacements, $q_{1-6}$. The strain in the adhesive at the current, $n+1$, increment is written as a function of $x$ and $q_{1-6}$ :

$$
\epsilon^{n+1}=f\left(q_{1-6}, x\right)
$$

while the displacements from the previous increment are used to define the adhesive strain at the previous increment, $\epsilon^{n}$, as a function of $x$ only:

$$
\epsilon^{n}=f(x)
$$

The energy is then minimized, which yields the $i^{\text {th }}, j^{\text {th }}$ component of the contribution to local joint stiffness matrix from the adhesive, $k$, to be:

$$
k_{i, j}=\frac{\partial^{2}}{\partial q_{i} \partial q_{j}} \int_{V_{a}} \frac{1}{2} \frac{d g\left(\epsilon^{n}\right)}{d \epsilon}\left(\epsilon^{n+1}\right)^{2} d V
$$

and the $i^{\text {th }}$ component contribution of the adhesive to the local joint force vector, $f$, to be

$$
f_{i}=\frac{\partial^{2}}{\partial q_{i}} \int_{V_{a}}\left(-g\left(\epsilon^{n}\right)+\frac{d g\left(\epsilon^{n}\right)}{d \epsilon}\left(\epsilon^{n}\right)\right) \epsilon^{n} d V
$$

The contribution of the adhesive to the local force vector and stiffness matrix can be added to the contributions of the two adherends. Since only the adhesive is modeled as nonlinear, the contributions of the adherends to the stiffness matrix are the same as in the linear case. The local stiffness matrix and local force vector can be used to find the local nodal displacements, $\{q\}$ :

$$
[k]\{q\}=\{f\} .
$$




\section{F. Assemble Global Matrices, Apply Loading, and Solve Global Equations}

Once the joint element stiffness matrix and load vector are found, they are assembled with the rest of the elements in the model. The loading increment is applied, and the system of linear equations is solved. For this particular study, an in-house finite element code was used to assemble and solve the finite element global equations. Future work will implement the method into an Abaqus ${ }^{11}$ user element to be integrated in global, vehicle scale models.

\section{G. Check for Adhesive Failure}

Once the nodal displacements are found, Eq. (2a) and (2b) are used to find the strain in the adhesive as a function of horizontal position, $x$. Then, a strain-based failure criterion based on the failure strain, $\epsilon_{\text {fail }}$, is used to determine if and how much the adhesive has failed (Fig. 6).

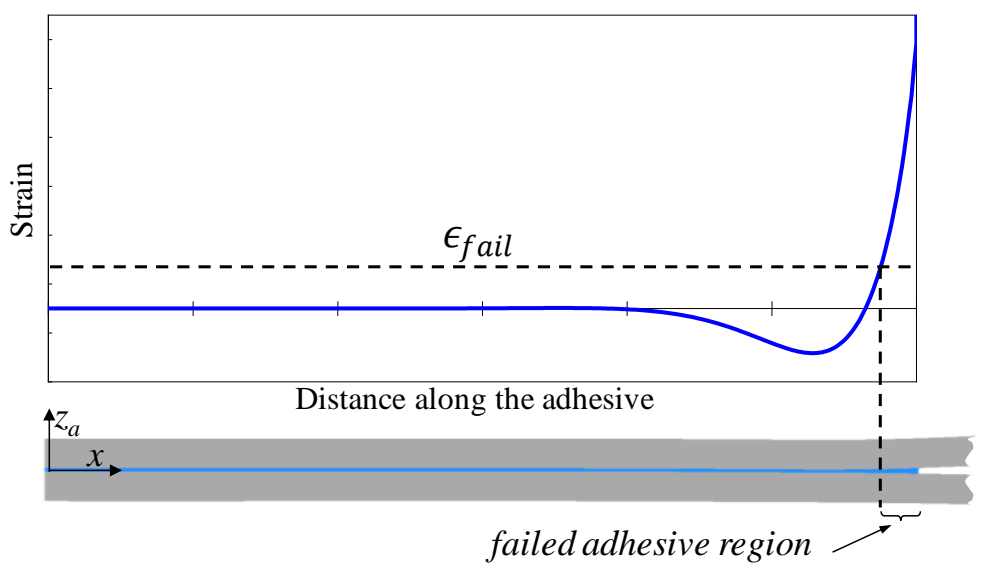

Figure 6. Example of modeling failure in the adhesive: the normal strain surpasses the failure strain at the right end of the adhesive, causing this region to be categorized as "failed".

\section{Beam Elements}
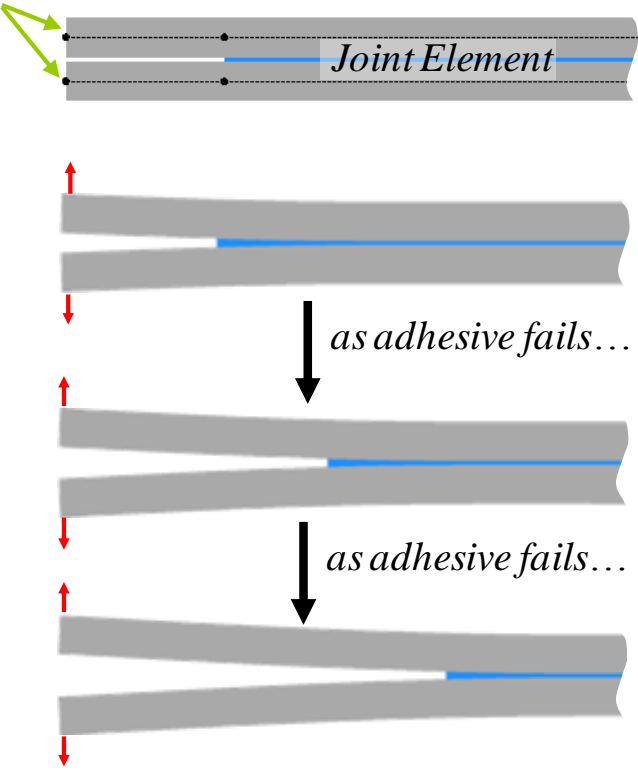

Figure 7. When failure occurs in the adhesive, the joint element is shortened and the adjacent beam elements are lengthened (DCB geometry assumed).
If failure is detected, the joint element is shortened by the length of the failed adhesive region, and the adjacent beam elements are lengthened to compensate (Fig. 7), with no continuity of displacements imposed between them. After this step is completed, the steps described in Section II A-G are repeated until the joint reaches equilibrium. After equilibrium is reached and further failure no longer occurs, the load is increased by one increment, and the process is repeated. Through this method, the stresses, strains, loads, and displacements for the joint can be found at each load increment as the joint deforms non-linearly and progressively fails.

\section{Comparison with Published Results}

The ability of the joint finite element to predict the force vs. displacement behavior was assessed through comparison with experimental and finite element results published by Song and Waas ${ }^{15}$. Results for a double cantilever beam (DCB) were chosen rather than a single lap joint because of the simple loading in the adhesive (no shear stress). This particular data set was chosen because the authors stated that the failure was fully interlaminar and cohesive, which is the type of failure currently modeled by the joint element.

Figure 8a shows a DCB specimen and the geometric 
parameters. The DCB specimens were constructed from two different 48 ply unidirectional composite laminates, E719/IM7 and E7T1/G40. The adhesive was one of the interlaminar matrix layers. The non-linear stress/strain relation of the adhesive was based on $\mathrm{G}_{\mathrm{IC}}$ and $\mathrm{E}_{\mathrm{a}}$ (Fig. 9). Normally, the mode one critical stress is preferred rather than the modulus, but this value was not provided by the authors. The geometric and material properties of the specimens are shown in Table 1. During the test, the loading was halted and the specimen was unloaded several times to measure the crack length within the specimen. The DCB specimen was modeled using one joint finite element accompanied by 2 beam elements on the top and bottom of the joint as shown in Fig. 8b.

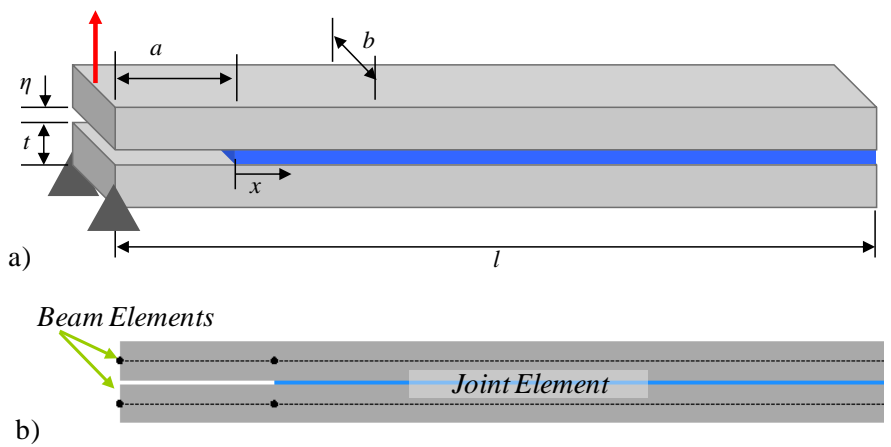

Figure 8. A typical DCB specimen, a) the geometric parameters and boundary conditions for the DCB specimens and $b)$ the mesh for the joint element DCB model.

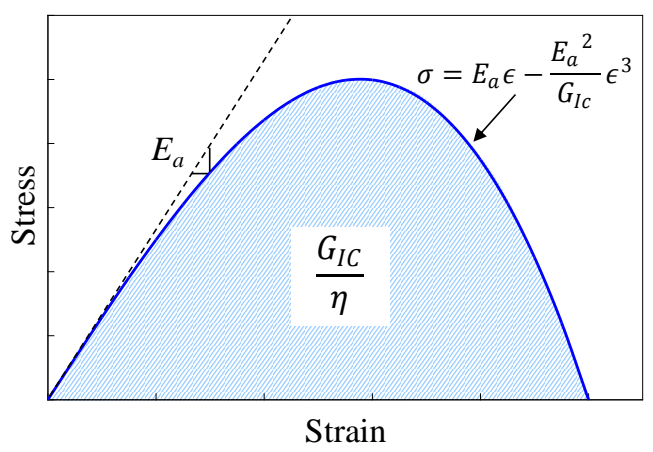

Figure 9. The stress/strain relation of the adhesive was defined based on the mode one energy release rate and modulus.

Table 1. Material properties and geometric parameters for Song/Waas ${ }^{15}$ DCB specimens.

\begin{tabular}{lcccccccc}
\hline \multicolumn{1}{c}{ Specimen } & \multicolumn{2}{c}{ Adhesive } & Adherend & \multicolumn{3}{c}{ Geometric Parameters } \\
& $\boldsymbol{E}_{\boldsymbol{a}}$ & $\boldsymbol{G}_{\boldsymbol{I c}}$ & $\boldsymbol{E}$ & $\boldsymbol{l}$ & $\boldsymbol{a}$ & $\boldsymbol{b}$ & $\boldsymbol{t}$ & $\boldsymbol{\eta}$ \\
& $(\mathbf{G P a})$ & $(\mathbf{N} / \mathbf{m})$ & $(\mathbf{G P a})$ & $(\mathbf{m m})$ & $(\mathbf{m m})$ & $(\mathbf{m m})$ & $(\mathbf{m m})$ & $(\boldsymbol{\mu m})$ \\
\hline E7T1/G40 & 4.1 & 335 & 116 & 200 & 52.6 & 15.5 & 4.65 & 35 \\
E719/IM7 & 3.3 & 1130 & 135 & 200 & 35.5 & 15.1 & 3.23 & 6 \\
\hline
\end{tabular}

Figure 10 compares the experimental force/displacement responses for the E719/IM7 DCB specimen acquired by Song and Waas ${ }^{15}$, along with the response predicted by these authors using an FEA model including discrete cohesive zone elements and the present joint element model. As can be seen, the present model was quite accurate at predicting the progressive failure of the joint based on the given material properties and parameters. The two models used different adhesive stress/strain relations, which probably accounts for the difference in predicted responses. However, Song and Waas ${ }^{15}$ reported that their FEA took 1-2 hours to run each load loop, while the present analysis took only 30-60 seconds per load loop, a speed increase of a factor of 120 . This demonstrates the usefulness of the joint finite element in performing quick, computationally efficient analysis of joints, even in progressive failure scenarios.

The plots in Fig. 11 are helpful in visualizing how the stress and strain evolve in the adhesive with increasing end displacement. Figure 11a shows the load/displacement plot for the E719/IM7 DCB specimen. The circles show points during loading at which stress (Fig. 11b) and strain (Fig. 11c) snapshots were taken. The solid circles correspond to solid lines on the stress and strain plots, while the hollow circles correspond to the dotted lines on the plots. The stress in the adhesive rises at the left end of the adhesive until the maximum stress according to the stress/strain relation (Fig. 9) is reached, at which point the stress at the end of the adhesive reduces to zero. At this point, the adhesive starts to fail. The strain reaches a critical level, about $1.6 \%$ strain, before failure begins. It can be seen that the shape of the stress/strain relation will have a significant effect on the stress levels at the end of the joint. 


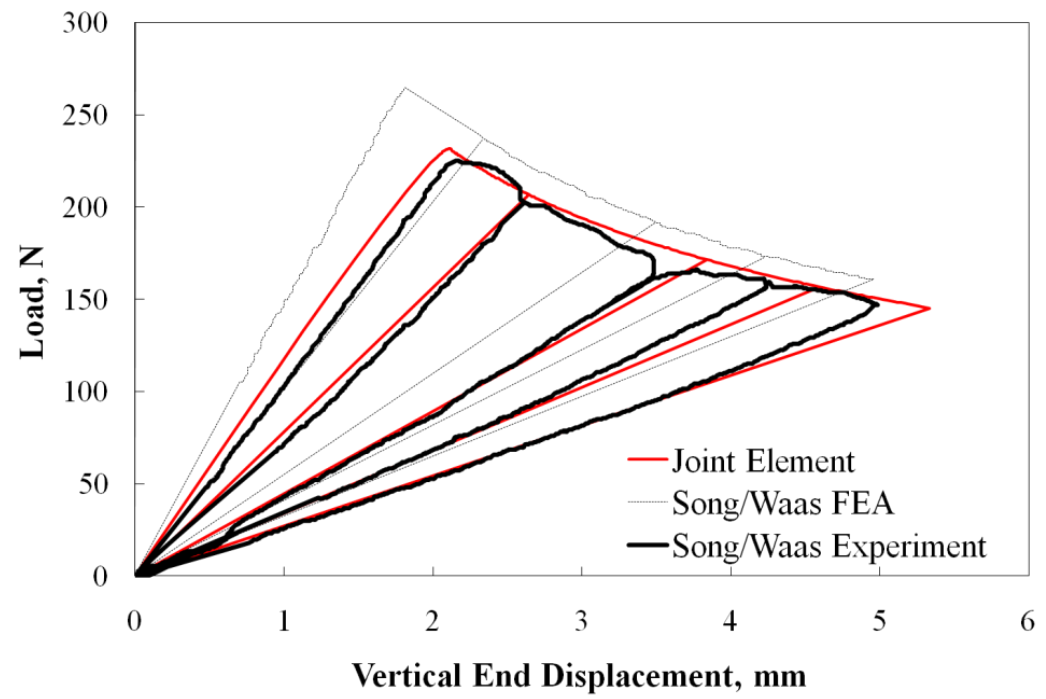

Figure 10. Load vs. displacement curves for E719/IM7 DCB specimen tested by Song and Waas ${ }^{15}$, along with their Finite Element Model and the present joint element model.

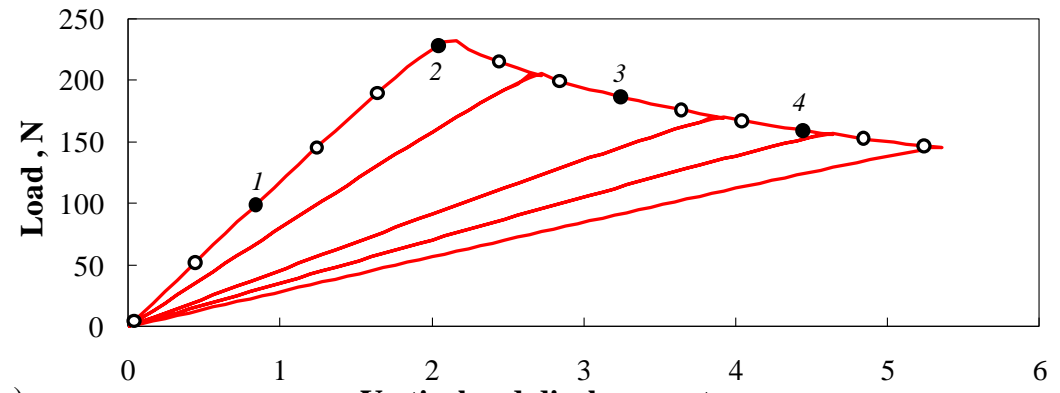

a)

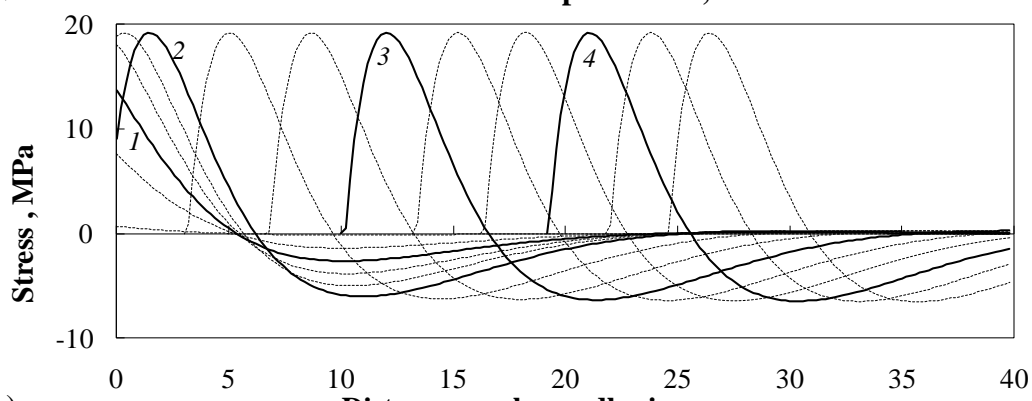

b)

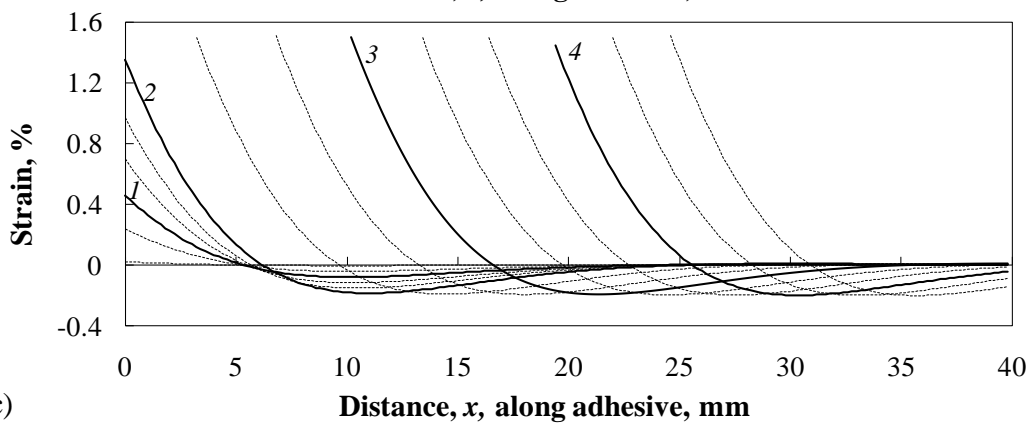

Figure 11. The solid and hollow circles in the a) load/displacement plot for the E719/IM7 DCB specimens correspond to the solid and dotted curves in the b) stress and c) strain plots. 
The behavior of the E7T1/G40 DCB specimens, shown in Fig. 12, was not predicted as accurately by the joint element model. The joint element model predicted nearly the same elastic slope as the Song/Waas FEA model, except with a more conservative prediction of the maximum load reached. Song/Waas used an interatomic force law to get the constitutive relations, while the present model used the adhesive modulus and critical strain energy release rate. It appears that for this case, the two methods resulted in very similar predicted responses. This comparison shows that the joint element can compete with discrete cohesive zone models in predicting the behavior of DCB joints.

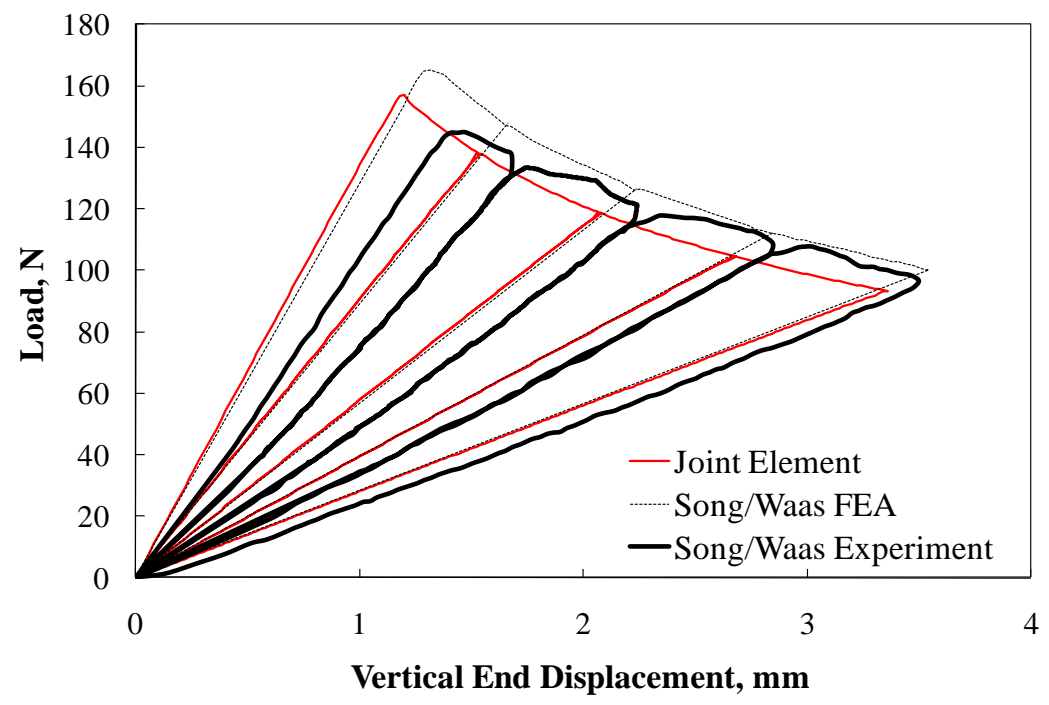

Figure 12. Load vs. displacement curves for E7T1/G40 DCB specimen tested by Song and Waas ${ }^{15}$, along with their Finite Element Model and the present joint element model.

\section{Comparison with Experimental Results}

DCB specimens were manufactured and tested to compare two paste adhesives, EA 9394 and EA 9309.3NA. The results of these tests were used to assess the ability of the joint finite element to predict the difference in performance of two adhesives with very different constitutive relations based on material properties obtained from tensile tests. Solid cylindrical specimens with a $3.175 \mathrm{~mm}$ diameter and $3.175 \mathrm{~mm}$ long test section were machined out of cast adhesive cylinders. The specimens were tested at NASA Glenn, and digital image correlation (DIC) techniques (Fig. 13a) were utilized to obtain the axial strain of the specimen at different loads. The strains for several points in the gauge section were averaged, and the stress was found by assuming constant stress in the cross section. Figure 13b shows characteristic stress/strain data for the two adhesives, and the equations used to fit the data with a curve. The material parameters used in the equations are found in Table 2 . The functions were chosen because they result in the same curve in compression and tension and seem to fit the data adequately. 


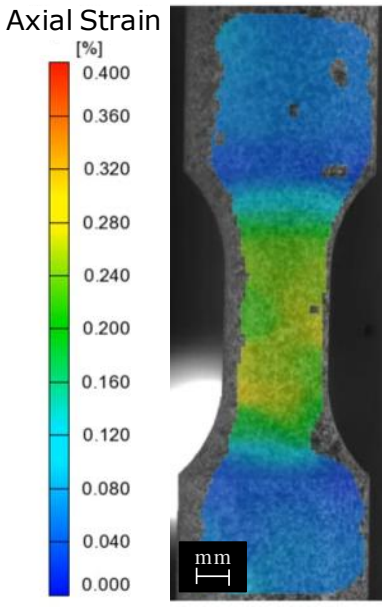

a)

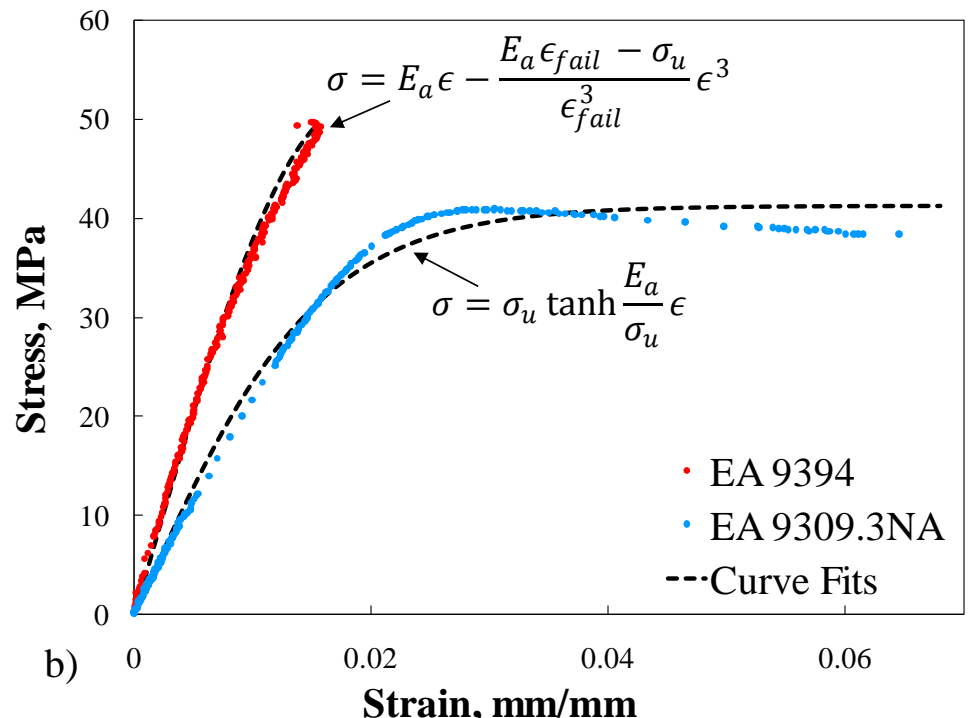

Figure 13. Using a) cylindrical tensile specimens and digital image correlation, the b) stress/strain relations of the adhesives EA 9394 and EA 9309.3NA could be defined by curve-fitting experimental data.

Table 2. Material properties and geometric parameters of DCB specimens.

\begin{tabular}{lcccccccccc}
\hline \multicolumn{1}{c}{} & \multicolumn{3}{c}{ Adhesive } & \multicolumn{3}{c}{ Adherend } & \multicolumn{3}{c}{ Geometric Parameters } \\
& $\boldsymbol{E}_{\boldsymbol{a}}$ & $\boldsymbol{\sigma}_{\boldsymbol{u}}$ & $\boldsymbol{\varepsilon}_{\text {fail }}$ & $\boldsymbol{v}$ & $\boldsymbol{E}$ & $\boldsymbol{l}$ & $\boldsymbol{a}$ & $\boldsymbol{b}$ & $\boldsymbol{t}$ & $\boldsymbol{\eta}$ \\
$(\mathbf{G P a})$ & $(\mathbf{M P a})$ & & & $(\mathbf{G P a})$ & $(\mathbf{m m})$ & $(\mathbf{m m})$ & $(\mathbf{m m})$ & $(\mathbf{m m})$ & $(\mathbf{m m})$ \\
\hline EA 9394 & 4.2 & 49.6 & 0.016 & 0.4 & 69 & 152.4 & 63.5 & 25.4 & 12.7 & 0.6 \\
EA 9309.3NA & 2.7 & 41.3 & 0.068 & 0.42 & 69 & 152.4 & 63.5 & 25.4 & 12.7 & 0.55 \\
\hline
\end{tabular}

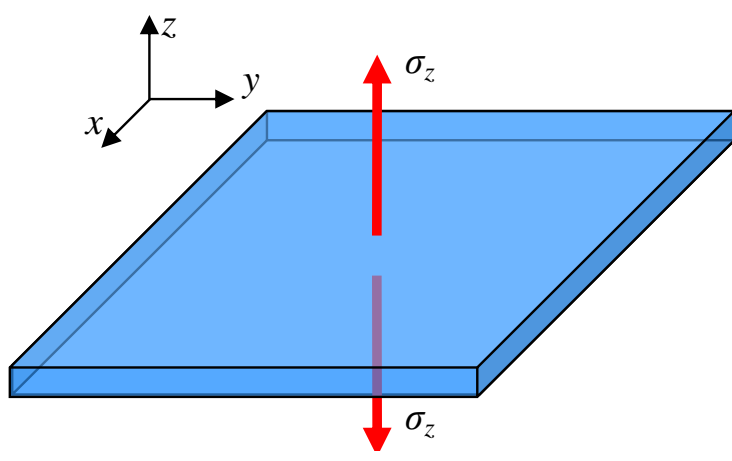

Figure 14. A thin adhesive layer with an applied stress is in a state of triaxial stress.
The tensile loading of a thin adhesive layer with relatively large in-plane dimensions differs greatly to that of a solid cylindrical specimen because the adhesive layer is extremely thin in one direction, and constrained from lateral displacement by the top and bottom adherends. Because of these conditions, the adhesive is effectively a body in plane strain in the two directions perpendicular to the adhesive thickness (Fig. 14). The adhesive is constrained from contracting (Poisson's effect) in the $x$ and $y$-directions while being loaded in the $z$-direction, which induces a stress in all three directions, commonly called a state of triaxial stress ${ }^{16}$. To find the stress/strain relation for a material under triaxial stress, consider first an isotropic, linearly elastic material. The normal stress in the $z$-direction is:

$$
\sigma_{z}=\frac{E}{1+v}\left[\epsilon_{z}+\frac{v}{1-2 v}\left(\epsilon_{z}+\epsilon_{x}+\epsilon_{y}\right)\right]
$$

The adhesive can be assumed to be in a state of plane stress in the $x y$-plane, and the strains $\epsilon_{x}$ and $\epsilon_{y}$ can be set to zero. Then, the normal stress in the $z$-direction reduces to: 


$$
\sigma_{z}=\frac{1-v}{(1+v)(1-2 v)} E \epsilon_{z}
$$

This shows that the effective "resistance" to deformation in the $z$-direction is amplified by a factor that depends on Poisson's ratio. Although this relation is intended for linear elasticity, the relation was assumed to hold for the nonlinear stress/strain relation. Therefore, the stress/strain relation was redefined as:

$$
\sigma=\frac{1-v}{(1+v)(1-2 v)} g(\epsilon),
$$

which effectively increases the adhesive modulus. Since the joint element considers only failure in the adhesive (cohesive failure), care was taken to ensure that the interface between the adherends and adhesive of the DCB specimens wouldn't be the point of failure. The adherends were 7071 T6 Aluminum, and the surfaces to be bonded were sanded, etched in lye, and anodized in a sulfuric acid solution prior to bonding. This treatment was sufficient to produce failures in the adhesive layer, as can be seen on the failure surfaces of a post-mortem specimen in Fig. 15. The failed specimen has adhesive covering both adherends, which means that the interface was not the plane of failure. Glass beads were used to maintain a consistent bond line thickness throughout the specimen, and pressure was applied to the specimen during curing. The specimens were allowed to cure for seven days at room temperature.

Three DCB specimens for each adhesive were tested on an Instron machine at $0.5 \mathrm{~mm} / \mathrm{min}$. All specimens failed cohesively like the specimen in Fig. 15. The force/displacement curves for all six specimens are shown in Fig. 16. The high strain-to-failure of EA 9309.3NA caused these specimens to hold over two times the amount of load as the EA 9394 specimens. The EA 9309.3NA specimens held so much load that the aluminum adherends deformed plastically before the adhesive failed. This can be seen in the plot as a gradual rounding prior to a drop in load. The EA 9394 specimens exhibited a load plateau rather than dropping in load after adhesive failure was initiated. It is possible that air bubbles in the adhesive caused the adhesive to fail prematurely, allowing the joint to not drop in load carrying capacity after failure initiated.

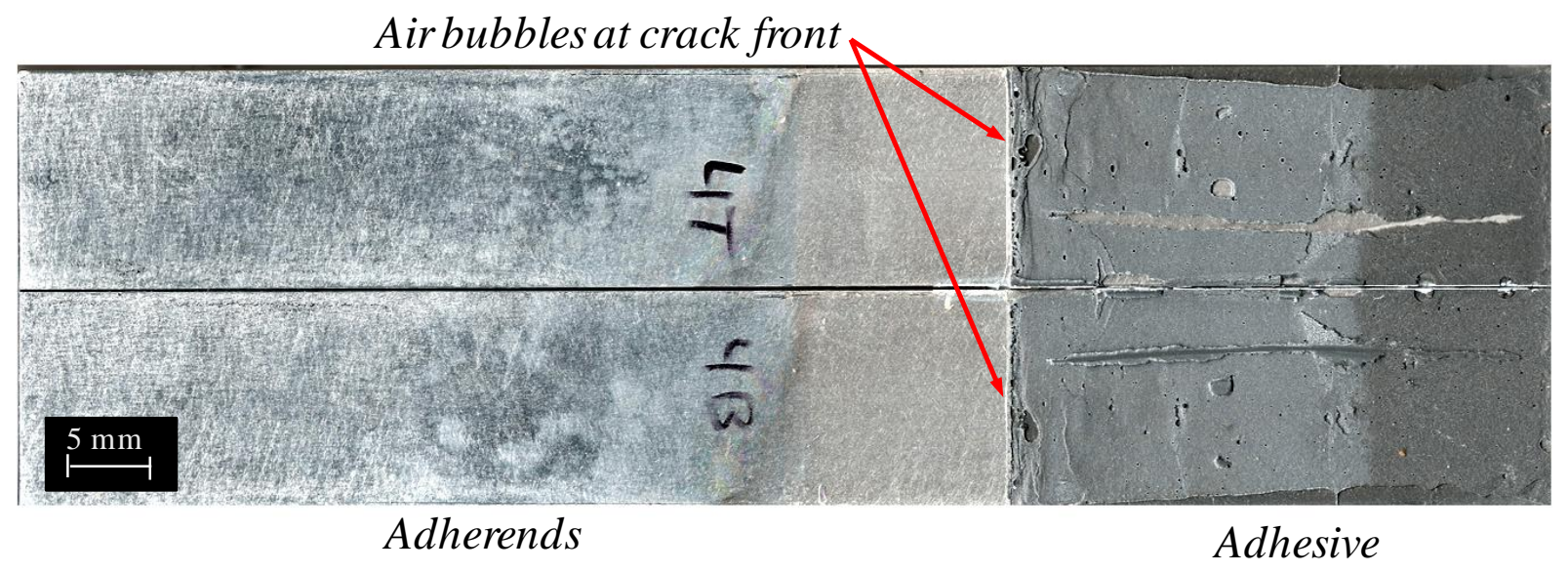

Figure 15. Two adherends of an EA 9394 DCB specimen after complete failure. The fact that adhesive is found on both adherends shows that failure occurred within the adhesive layer as desired.

The joint element model was able to capture the behavior of the joints rather well. It was found that compliance in the experimental load train caused the models to over-predict even the initial linear portion of the loading. To compensate for this system compliance, the length of the adherends was lengthened by $7.5 \%$ for both DCB specimen types. This number was determined by fitting a linearly elastic model to the initial portion of the experimental force/displacement plot. While not exactly matching the experimental data, the model was rather accurate considering the aforementioned adherend plasticity and air bubbles in the end of the adhesive. These experiments were very effective in displaying the ability of the joint element to predict failure, along with showing how constitutive relations can be applied to get progressive failure of a thin adhesive layer.

14

American Institute of Aeronautics and Astronautics 092407 


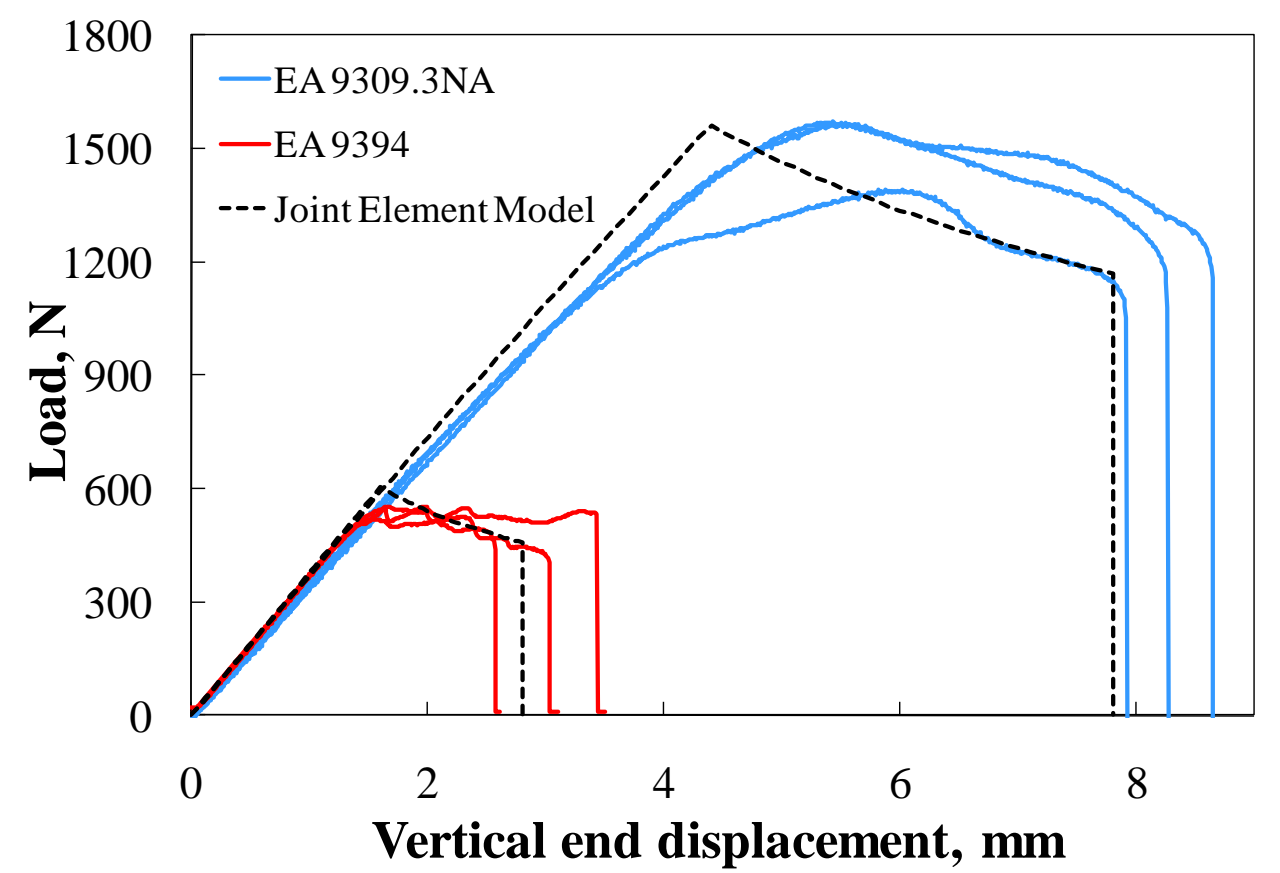

Figure 16. Load vs. displacement curves for DCB specimens with aluminum adherends and EA 9394 and EA 9309.3NA adhesive, along with the joint finite element model prediction.

\section{Conclusion}

This study showed how a single finite element could be used to capture the behavior of an entire joint by using an analytical method to solve for the appropriate shape functions rather than prescribe the shape functions using a simple polynomial. Additionally, progressive failure was included by defining the stress as a non-linear function of the strain and enforcing a strain-based failure criterion. Failure of the adhesive was approximated by shortening the joint element by the length of the failed adhesive and lengthening the adjoining beam elements. The accuracy of the joint element model was assessed through comparison with finite element and experimental DCB test results published by Song and Waas ${ }^{15}$, and experimental DCB tests conducted by the authors. Results showed that the joint element model could capture the force/displacement behavior of the joint quite well, with a significant computational efficiency advantage compared to a traditional FEA. The assessment also demonstrated how the stress/strain relation of the adhesive could be obtained using fracture mechanics or constitutive relations determined by bulk tensile tests. When using tensile tests, a factor was used to amplify the stress/strain relation to account for additional deformation resistance due to a triaxial state of stress. With proper implementation of this method, a single finite element can be used in a broader, global-scale model to capture the behavior and failure of joints.

\section{Acknowledgements}

The authors would like to thank Justin Bail of the Ohio Aerospace Institute for testing the adhesive tensile specimens at NASA Glenn. Portions of this work were financially supported by the Space Vehicle Technology Institute under grant NCC3-989 jointly funded by NASA and the Department of Defense. Additional financial support was provided by NASA Glenn Research Center through the GSRP Fellowship.

\section{References}

${ }^{1}$ Hart-Smith, L., "Adhesive Bonding of Composite Structures- Progress to Date and Some Remaining Challenges,” Journal of Composites Technology and Research, Vol. 24, No. 3, 2002, pp. 133-151.

${ }^{2}$ Frostig, Y., Thomsen, O., Mortensen, F., "Analysis of Adhesive-Bonded Joints, Square-End, and Spew-Fillet- High-Order Theory Approach," Journal of Engineering Mechanics, Vol. 125, No. 11, 1999, pp. 1298-1307.

${ }^{3}$ Gustafson, P. A. and Waas, T. A. "A Macroscopic Finite Element for a Symmetric Double-lap Joint Subjected to Mechanical and Thermal Loading," Proceedings of the AIAA/ASME/ASCE/AHS/ASC 48 ${ }^{\text {th }}$ SDM Conference, April 23-26, 2007, Honolulu, HI, No. 2007-2308, American Institute of Aeronautics and Astronautics, 2007. 
${ }^{4}$ Volkersen, O., "Die Nietkraftverteilung in Zugbeanspruchten mit Konstanten Laschenquerschritten," Luftfahrtforschung, Vol 15, 1938, pp. 41-47.

${ }^{5}$ Goland, M. and Reissner, E. "The Stresses in Cemented joints," Journal of Applied Mechanics, Vol. 11, 1944, pp A17-A27.

${ }^{6}$ Hart-Smith, L. J. "Adhesive bonded single lap joints," Tch. Rep. NASA CR 112236, Douglas Aircraft, McDonnel Douglas Corporation, Long Beach, California, 1973.

${ }^{7}$ Mortensen, F. and Thomsen, O.T., "Analysis of Adhesive Bonded Joints: a Unified Approach," Composites Science and Technology, Vol. 62, 2002, pp. 1011-1031.

${ }^{8}$ Delale, F., Erdogan, F., and Aydinoglu, M. N., "Stresses in Adhesively Bonded Joints: A Closed-Form Solution," Journal of Composite Materials, Vol. 15, 1981, pp. 249-271.

${ }^{9}$ Zhang, J, Bednarcyk, B. A., Collier, C, Yarrington, P., Bansal, Y., and Pindera, M. J., "3D Stress Analysis of Adhesively Bonded Composite Joints," Proceedings of the AIAA/ASME/ASCE/AHS/ASC 46 ${ }^{\text {th }}$ SDMConference, April 18-21 2005, Austin, Texas, No. 2005-2021, American Institute of Aeronautics and Astronautics, 2005.

${ }^{10}$ Stapleton, S. E. and Waas, A. M., "Macroscopic Finite Element for a Single Lap Joint," Proceedings of the AIAA/ASME/ASCE/AHS/ASC 50 ${ }^{\text {th }}$ SDM Conference, May 4-7 2009, Palm Springs, California, No. 2009-2449, American Institute of Aeronautics and Astronautics, 2009.

${ }^{11}$ ABAQUS, Inc, ABAQUS user Manuel v6.7-1, Electronic Version, 2006.

${ }^{12}$ Tsai, M. Y. and Morton, J., "An evaluation of Analytical and Numerical Solutions to the Single-Lap Joint," Int. J. Solids Structures, Vol. 31, No. 18, 1994, pp. 2537-2563.

${ }^{13}$ Chen, C., Linear System Theory and Design, $3^{\text {rd }}$ ed., Oxford University Press, New York, 1999.

${ }^{14}$ Molder, C. and Van Loan, C., "Nineteen Dubious Ways to Compute the Exponential of a Matrix, Twenty-Five Years Later", SIAM Review, Vol. 45, No. 1, pp 3-000.

${ }^{15}$ Song, S.J., and Waas, A.M., "Mode I Failure of Laminated Polymeric Composites," Engineering Fracture Mechanics, Vol. 49, No. 1, 1994, pp 17-27.

${ }^{16}$ Sechler, E., Elasticity in Engineering, Wiley: Chapman \& Hall, New York, 1952. 